We introduce a numerical procedure for the construction of interpolation and quadrature formulae on bounded convex regions in the plane. The construction is based on the behavior of spectra of certain multiplication operators and leads to nodes which are inside a prescribed convex region in $\mathbb{R}^{2}$. The resulting interpolation schemes are numerically stable and the quadrature formulae have positive weights and almost (but not quite) optimal numbers of nodes. The performance of the algorithm is illustrated by several numerical examples.

\title{
Spectra of Multiplication Operators as a Numerical Tool
}

\author{
B. Vioreanu ${ }^{\dagger}$ and V. Rokhlin $\ddagger$ \\ Technical Report YALEU/DCS/TR-1443 \\ March 3, 2011
}

† Dept. of Mathematics, Yale University, New Haven CT 06511.

¥ Dept. of Mathematics and Computer Science, Yale University, New Haven CT 06511

The authors were supported in part by the U.S. DoD under ONR Grants \#N00014-101-0570 and \#N00014-09-1-0108, and AFOSR Grant \#FA9550-09-1-0241.

Approved for public release: distribution is unlimited.

Keywords: Multiplication operator, interpolation, numerical integration. 


\section{INTRODUCTION}

Numerical integration and interpolation constitute one of oldest areas of numerical analysis, and find applications in most branches of applied mathematics. In general outline, the formulation is as follows. Given a region $\Omega \in \mathbb{R}^{k}$ (usually, with $k \leq 3$ ) and a collection of functions $\varphi_{1}, \varphi_{2}, \ldots, \varphi_{n}$ mapping $\Omega$ to $\mathbb{R}^{1}$, we would like to find a collection of points $x_{1}, x_{2}, \ldots, x_{m}$ such that for each point $x$ in $\Omega$, there exist coefficients $\alpha_{1}(x), \alpha_{2}(x), \ldots, \alpha_{m}(x)$ such that

$$
f(x)=\sum_{j=1}^{m} \alpha_{j} f\left(x_{j}\right),
$$

for all $f: \Omega \rightarrow \mathbb{R}^{1}$ of the form

$$
f(x)=\sum_{i=1}^{n} \beta_{i} \varphi_{i}(x) ;
$$

in other words, the interpolation formula (1.1) is exact for any $f$ that is a linear combination of the functions $\left\{\varphi_{i}\right\}$.

In practice, (1.1) is not sufficient, since if the resulting interpolation formulae are to be of any practical use, the coefficients $\left\{\alpha_{i}\right\}$ must not be very large, and have to be easy to evaluate. The following theorem (see [9]) guarantees the existence of numerically acceptable interpolation coefficients under extremely weak conditions.

Theorem 1.1. Suppose that $S$ is an arbitrary set, $n$ is a positive integer, $f_{1}, f_{2}, \ldots, f_{n}$ are bounded complex-valued functions on $S$, and $\varepsilon$ is a positive real number such that $\varepsilon \leq 1$. Then, there exist $n$ points $x_{1}, x_{2}, \ldots, x_{n}$ in $S$ and $n$ functions $g_{1}, g_{2}, \ldots, g_{n}$ on $S$ such that

$$
\left|g_{k}(x)\right| \leq 1+\varepsilon
$$

for all $x$ in $S$ and $k=1,2, \ldots, n$, and

$$
f(x)=\sum_{k=1}^{n} f\left(x_{k}\right) g_{k}(x)
$$

for all $x$ in $S$ and any function $f$ defined on $S$ via the formula

$$
f(x)=\sum_{i=1}^{n} c_{i} f_{i}(x),
$$

for some complex numbers $c_{1}, c_{2}, \ldots, c_{n}$.

Thus, the existence of a stable interpolation formula is never a serious issue; this paper deals with numerical procedures for constructing such interpolation formulae.

Numerical integration of functions is closely related to their numerical interpolation, but involves a number of subtleties. Given a region $\Omega \in \mathbb{R}^{k}$ and a collection of functions 
$\varphi_{1}, \varphi_{2}, \ldots, \varphi_{n}: \Omega \rightarrow \mathbb{R}^{1}$, one wishes to find points $x_{1}, x_{2}, \ldots, x_{m} \in \Omega$ and coefficients $w_{1}, w_{2}, \ldots, w_{m} \in \mathbb{R}^{1}$ such that for each of the functions $\left\{\varphi_{i}\right\}$,

$$
\int_{\Omega} \varphi_{i}(x) d x=\sum_{j=1}^{m} w_{j} \varphi_{i}\left(x_{j}\right) ;
$$

the pair $\left\{\left\{x_{i}\right\},\left\{w_{i}\right\}\right\}$, is frequently referred to as a quadrature formula for the functions $\left\{\varphi_{i}\right\}$, with $\left\{x_{i}\right\}$ the nodes, and $\left\{w_{i}\right\}$ the weights of the quadrature.

Again, (1.6) is not sufficient for the quadrature $\left\{\left\{x_{i}\right\},\left\{w_{i}\right\}\right\}$ to be of practical use. Indeed, its numerical stability will be poor if the weights $\left\{w_{i}\right\}$ are large. Obviously, if all of the weights $\left\{w_{i}\right\}$ are positive, this issue does not arise, and quadratures with positive weights are generally preferred (though in most cases not absolutely necessary).

Obviously, given an interpolation formula of the form (1.4) for a set of functions $f_{1}, f_{2}, \ldots, f_{n}$, it is easy to construct a quadrature that is numerically stable; this observation is formalized in the following corollary to Theorem 1.1 (see [9]).

Corollary 1.1. Suppose that $S$ is a measure space, $w$ is a nonnegative real-valued integrable function on $S$ (that serves as the weight for integration), $n$ is a positive integer, $f_{1}, f_{2}, \ldots, f_{n}$ are bounded complex-valued integrable functions on $S$, and $\varepsilon \leq 1$ is a positive real number.

Then, there exist $n$ complex numbers $w_{1}, w_{2}, \ldots, w_{n}$ such that

$$
\left|w_{k}\right| \leq(1+\varepsilon) \int w(x) d x
$$

for all $k=1,2, \ldots, n$, and

$$
\int f(x) w(x) d x=\sum_{k=1}^{n} w_{k} f\left(x_{k}\right)
$$

for any function $f$ defined on $S$ via the formula (1.5), where $x_{1}, x_{2}, \ldots, x_{n}$ are the $n$ points in $S$ chosen in Theorem 1.1.

One fundamental difference between interpolation and integration is the fact that interpolation in an $n$-dimensional space always requires at least $n$ points (and, according to Theorem 1.1, $n$ points are always sufficient); on the other hand, a quadrature formula for integrating $n$ functions often requires fewer than $n$ points. The reader is referred to Section 2.4 for a somewhat more detailed discussion of this class of issues.

In one dimension, the existing theory of quadrature formulae is quite satisfactory. Gaussian quadratures (see, for instance, [3]) are optimal for integrating polynomials up to a fixed degree, while generalized Gaussian quadratures are used for integrating more general classes of functions including smooth functions and functions with end-point singularities $[8,12]$.

In dimensions higher than 1 , much less is known regarding optimal numerical integration formulae. Classical expositions on constructing quadratures for multiple integrals can be found in [4], [6], [7], and a more recent overview of the development of the theory is given in [2].

This paper is partly devoted to constructing quadratures on convex regions in two dimensions exhibiting Gaussian behavior (the number of nodes of the quadrature is 
smaller than the number of functions to be integrated) using a simple scheme, which is relatively easy to implement. The quadratures respect the symmetry of the region, have positive weights and all their nodes are interior to the region of integration.

The structure of this paper is as follows. Section 2 introduces numerical preliminaries. In Section 3 we develop the mathematical apparatus to be used by the algorithm in Section 4. We summarize the numerical results in Section 5.

\section{Numerical Preliminaries}

2.1. Singular Value Decomposition. The singular value decomposition (SVD) is a ubiquitous tool in numerical analysis. In the case of real matrices, the SVD is given by the following lemma (see, for instance, [5] for more details).

Lemma 2.1. For any $n \times m$ real matrix $A$, there exist a positive integer $p$, an $n \times p$ real matrix $U$ with orthonormal columns, an $m \times p$ real matrix $V$ with orthonormal columns, and a $p \times p$ real diagonal matrix $S=\left(s_{i j}\right)$ whose diagonal entries are positive, such that $A=U S V^{*}$ and $s_{i i} \geq s_{i+1, i+1}$ for all $i=1, \ldots, p-1$.

The diagonal entries $s_{i i}$ of $S$ are the nonzero singular values of $A$. The columns of $U$ span the column space of the matrix $A$ and hence provide an orthonormal basis of the column space of $A$. This property of the SVD is used in Section 4 for the computation of orthonormal basis functions.

2.2. The QR Method for finding the spectrum of a matrix. There are several methods available for determining the spectrum of a complex matrix (see, for example, [5]). In this paper, the QR method, as described in [10], is used for computing the eigenvalues of a complex linear operator on a finite-dimensional space.

2.3. Least Squares Newton's Method. Newton's method is a well-known numerical technique for solving equations of the form $F(x)=0$, where $F: \mathbb{R}^{m} \longrightarrow \mathbb{R}^{n}$ is a continuously differentiable function of the form

$$
F(x)=\left(\begin{array}{c}
f_{1}(x) \\
\vdots \\
f_{n}(x)
\end{array}\right)
$$

and $x=\left(x_{1}, \ldots, x_{m}\right)^{T}$. The method uses the Jacobian matrix $J$ of $F$, which is defined by the formula

$$
J(x)=\left(\begin{array}{ccc}
\frac{\partial f_{1}}{\partial x_{1}}(x) & \cdots & \frac{\partial f_{1}}{\partial x_{m}}(x) \\
\vdots & & \vdots \\
\frac{\partial f_{n}}{\partial x_{1}}(x) & \cdots & \frac{\partial f_{n}}{\partial x_{m}}(x)
\end{array}\right),
$$

and its pseudoinverse, which is denoted by $J^{\dagger}(x)$. We illustrate the application of the method in the following theorem, under the constraints relevant to our paper. When $m \neq n$, the procedure is referred to as the least squares Newton's method [11]. 
Theorem 2.1. Suppose that $F: \mathbb{R}^{m} \longrightarrow \mathbb{R}^{n}(m \geq n)$ is a continuously differentiable function and $\xi \in \mathbb{R}^{m}$ satisfies the condition

$$
F(\xi)=0 .
$$

Furthermore, suppose that $J(x)$ defined by (2.2) has full row rank for all $x$ in an $\varepsilon$ neighborhood $n_{\varepsilon}(\xi)=\{x:\|x-\xi\|<\varepsilon\}$ of $\xi$. Let $\left(x_{k}\right)_{k \geq 0}$ be a sequence of vectors defined by the formula

$$
x_{k+1}=x_{k}-J^{\dagger}\left(x_{k}\right) F\left(x_{k}\right),
$$

and let $\left(h_{n}\right)_{n \geq 0}$ be the sequence of differences

$$
h_{n}=x_{n}-\xi .
$$

Then there exist $\tilde{\varepsilon}>0, \delta>0$ and a nonnegative integer $N_{\tilde{\varepsilon}, \delta}$ such that, if $\left\|x_{0}-\xi\right\|<\tilde{\varepsilon}$, then the inequality

$$
\left\|h_{n+1}\right\| \leq \delta\left\|h_{n}\right\|^{2}
$$

is true for all $k \geq N_{\tilde{\varepsilon}, \delta}$.

2.4. Quadratures. Quadrature rules are expressions of the form

$$
\sum_{i=1}^{n} w_{i} \varphi\left(x_{i}\right),
$$

where the points $x_{i} \in \mathbb{R}^{d}$ and the coefficients $w_{i} \in \mathbb{R}$ are referred to as the nodes and weights of the quadrature, respectively. They serve as approximations to integrals of the form

$$
\int_{\Omega} \varphi(x) w(x) d x
$$

where $w$ is a positive weight function and $\Omega \subset \mathbb{R}^{d}$ is the region of integration.

Quadratures are typically chosen so that the formula (2.7) is equal to the desired integral (2.8) for some set of functions, commonly polynomials of some fixed order. A quadrature which integrates exactly all polynomials of (total) degree at most $k$ and does not integrate exactly some polynomial of degree $k+1$ is said to have order $k$. For instance, Gaussian quadrature rules consist of $n$ nodes and have order $2 n-1$.

Given a weight function $w$, an integration region $\Omega$ and $m$ functions defined on $\Omega$, a classical problem in numerical integration is to determine the minimum number of nodes $n$ needed to integrate exactly all the $m$ functions, and to find quadratures which achieve that minimum. Such quadratures are called optimal. In one dimension, Gaussian quadratures are known to be optimal rules for integrating polynomials up to a fixed degree. In higher dimensions, however, the problem has not been solved [2].

Since each node $x \in \mathbb{R}^{d}$ is determined by $d$ parameters and one additional parameter is given by the weight corresponding to the node, an $n$-point quadrature in $\mathbb{R}^{d}$ is determined by $n(d+1)$ parameters. For this reason, it is natural to expect that an optimal quadrature in $d$ dimensions which integrates $m$ functions has $n=\left\lceil\frac{m}{d+1}\right\rceil$ nodes, where $\lceil x\rceil$ denotes the smallest integer greater or equal to $x$. Based on this observation, the authors of [11] introduce an efficiency coefficient $e$ to differentiate quadratures which 
integrate the same set of functions and have a different number of nodes. If $n$ is the number of nodes of the quadrature, $m$ is the number of functions which are integrated exactly, and $d$ is the dimension of the space, then the coefficient $e$ is defined as

$$
e=\frac{m}{n(d+1)} \text {. }
$$

For Gaussian quadratures in one dimension, $e$ is equal to 1. Quadratures having the coefficient $e$ close to 1 are called efficient.

2.5. Tensor Product Rules. An obvious way of obtaining quadratures in two dimensions is to combine two one-dimensional quadratures in the following way.

Let $R$ be an $m$-point quadrature over an interval $I$,

$$
R(f)=\sum_{i=1}^{m} w_{i} f\left(x_{i}\right) \approx \int_{I} f(x) d x
$$

and $S$ be an $n$-point quadrature over an interval $J$,

$$
S(f)=\sum_{j=1}^{m} v_{j} f\left(x_{j}\right) \approx \int_{J} f(x) d x .
$$

The tensor product of $R$ and $S$ is the $m n$-point quadrature rule over the rectangle $I \times J$ defined by

$$
R \times S(f)=\sum_{i=1}^{m} \sum_{j=1}^{n} w_{i} v_{j} f\left(x_{i}, y_{j}\right) \approx \int_{I \times J} f(x, y) d x d y .
$$

The following theorem follows from Fubini's Theorem; a proof can be found, for instance, in $[4]$.

Theorem 2.2. Let $R$ be a quadrature rule that integrates $f(x)$ exactly over the interval I and $S$ be a quadrature rule that integrates $g(y)$ exactly over the interval $J$. Furthermore, let $h(x, y)=f(x) g(y)$. Then the tensor product quadrature $R \times S$ integrates $h(x, y)$ exactly over $I \times J$.

Tensor product quadratures are relatively easy to construct, however, they are not optimal in terms of efficiency. For example, let us consider two $n$-point Gaussian quadratures on the interval $[-1,1]$, each of them of order $2 n-1$. Their tensor product is an $n^{2}$-point quadrature on the square $[-1,1] \times[-1,1]$ of order $2 n-1$ (all monomials $x^{i} y^{j}$ of total degree $i+j$ at most $2 n-1$ can be expressed as products of monomials in the variables $x$ and $y$ of degree at most $2 n-1$, hence they are integrated exactly by the tensor product; however, there are polynomials of degree $2 n$, for instance $x^{2 n}$, which are not integrated exactly by this quadrature). The number of monomials $x^{i} y^{j}$ of total degree at most $2 n-1$ is $n(2 n+1)$, therefore the efficiency coefficient of the tensor product of two Gaussian quadratures is

$$
e=\frac{n(2 n+1)}{3 n^{2}}=\frac{2 n+1}{3 n},
$$

quantity which approaches $\frac{2}{3}$ as $n$ increases. 


\section{Mathematical Apparatus}

The main result of this section is Theorem 3.2, which allows us to discretize bounded convex regions in the complex plane. It is based on the observation that all the eigenvalues of a certain linear operator on a finite-dimensional subspace of $L^{2}(X)$, where $X$ is a convex, closed and bounded set in the plane, fall within $X$. Our proof of Theorem 3.2 involves the geometrical result introduced in the following section.

\subsection{A geometrical lemma.}

Lemma 3.1. Let $X$ be a convex, closed and bounded subset of the complex plane and $\lambda \in \mathbb{C}$ be a point outside $X$. Then there exists a point $\mu \in \mathbb{C}$ such that $\mu$ lies outside $X$ and

$$
|\lambda-\mu|>|z-\mu|
$$

for all z in $X$.

Proof. Let us denote by $d$ the distance from $\lambda$ to the set $X$ :

$$
d=d(\lambda, X)=\inf _{z \in X}|z-\lambda|
$$

Since $X$ is closed, the infimum $d$ is attained for some $z_{\lambda}$ in $X$, and is positive because $\lambda$ is not in $X$ :

$$
d=\left|z_{\lambda}-\lambda\right|>0 .
$$

Consider the circle $C$ of radius $d$ centered at $\lambda$ and the tangent $l$ to $C$ at the point $z_{\lambda}$ (see Figure 1).

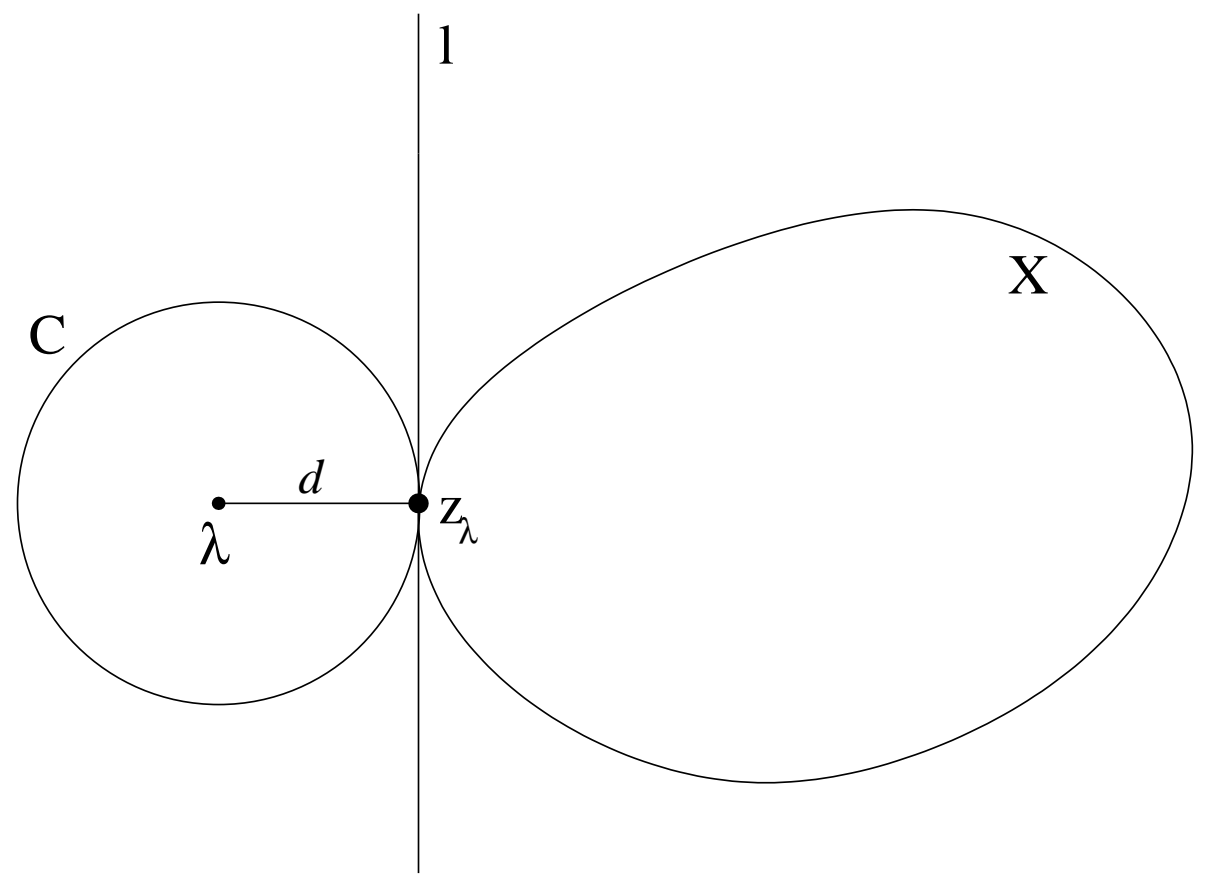

Figure 1 . The tangent $l$ to the circle $C$ centered at $\lambda$, at the point $z_{\lambda}$. The point $z_{\lambda}$ minimizes the distance from $\lambda$ to the set $X$. 
The line $l$ determines two half-planes: one containing the point $\lambda$ and one not containing the point $\lambda$. We observe that $X$ has no points inside the open half-plane determined by $l$ that contains the point $\lambda$.

Let us prove this observation by contradiction. To this end, suppose that there exists a point $z$ in $X$ which lies in the open half-plane containing $\lambda$. Since $z$ and $\lambda$ lie in the same half-plane determined by the tangent $l$, and $z$ is not on the line $l$, a part of the segment $\left[z z_{\lambda}\right]$ falls inside the circle $C$ (see Figure 2).

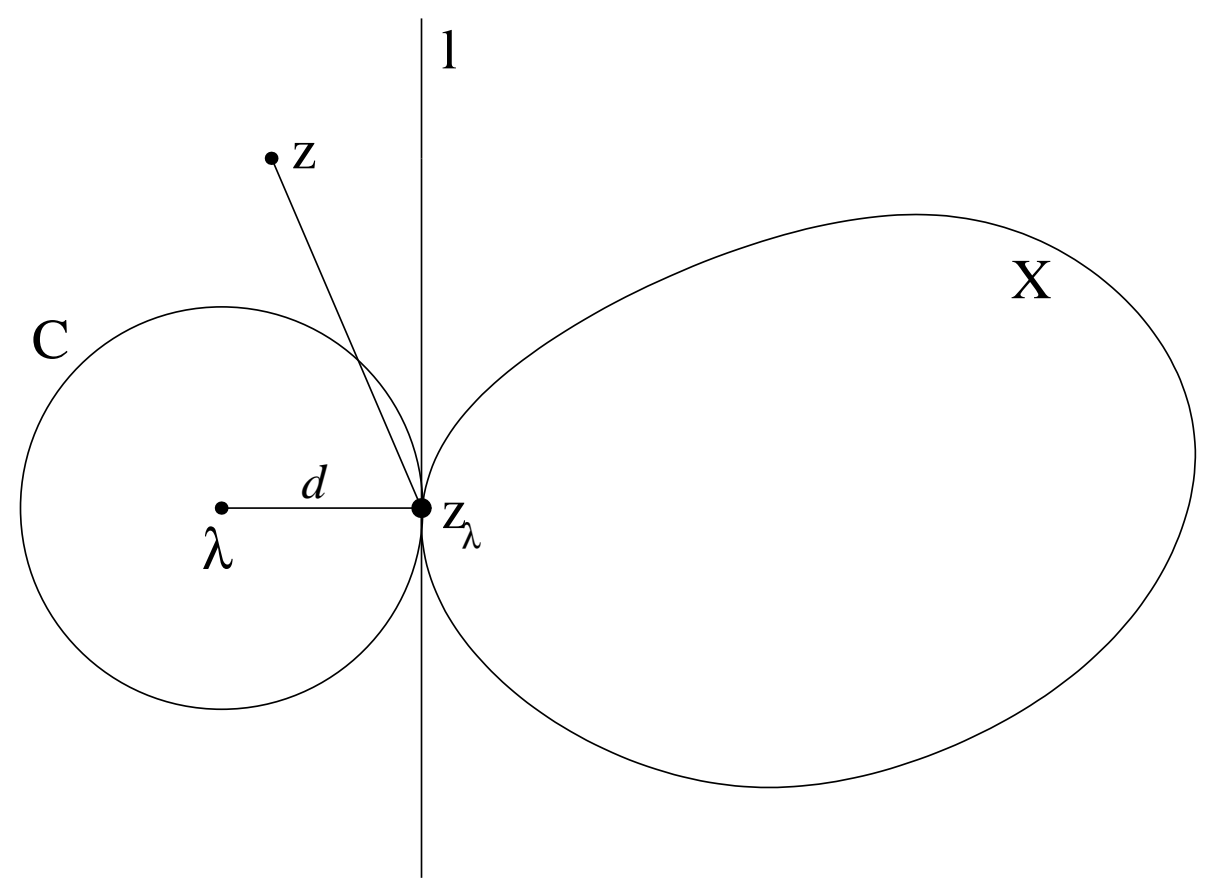

Figure 2. Proof of the observation by contradiction: if a point $z$ in $X$ lies in the open half-plane determined by the line $l$ which contains the point $\lambda$, then some points of $X$ fall inside the circle $C$.

However, $X$ is convex and the points $z$ and $z_{\lambda}$ lie in $X$, therefore the whole line segment $\left[z z_{\lambda}\right]$ is contained in $X$. Consequently, some points of $X$ fall inside the circle $C$, at distance less than $d$ to $\lambda$, contradicting our choice of $z_{\lambda}$. We conclude that the set $X$ is contained in the closed half-plane determined by $l$ that does not contain $\lambda$.

Since $X$ is bounded, we can find a square box $L_{1} L_{2} L_{3} L_{4}$ that contains $X$. Moreover, by the previous observation, we can choose the box such that the points $L_{1}, L_{2}$ lie on the line $l$ and the points $L_{3}, L_{4}$ lie in the half-plane determined by $l$ that does not contain $\lambda$. Finally, we can choose the square box big enough so that the length $s$ of one of its sides is greater than $d$ (see Figure 3 ).

Let $\mu$ be the point on the the half-line passing through the points $\lambda$ and $z_{\lambda}$ with initial point $\lambda$ with the following property:

$$
\left|z_{\lambda}-\mu\right|=\frac{s^{2}}{d}
$$

Since, by construction, $s>d$, multiplying by $\frac{s}{d}$ we obtain $\frac{s^{2}}{d}>s$, hence the point $\mu$ lies outside the square box $L_{1} L_{2} L_{3} L_{4}$ and therefore outside $X$ (see Figure 4 ). 


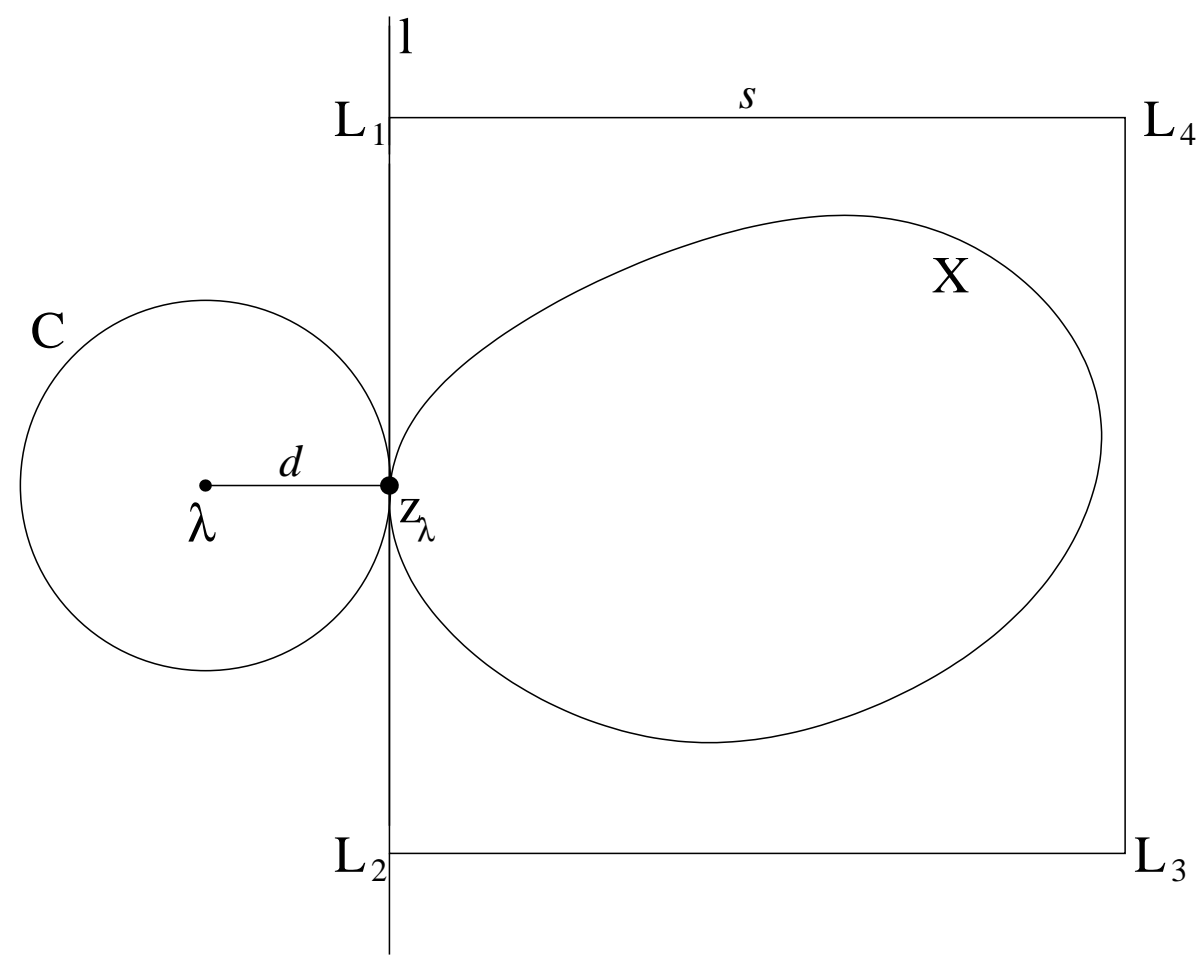

Figure 3. $X$ is enclosed in a square box $L_{1} L_{2} L_{3} L_{4}$ lying in the halfplane determined by $l$ that does not contain $\lambda$. The length $s$ of a side of the square is greater than $d$.

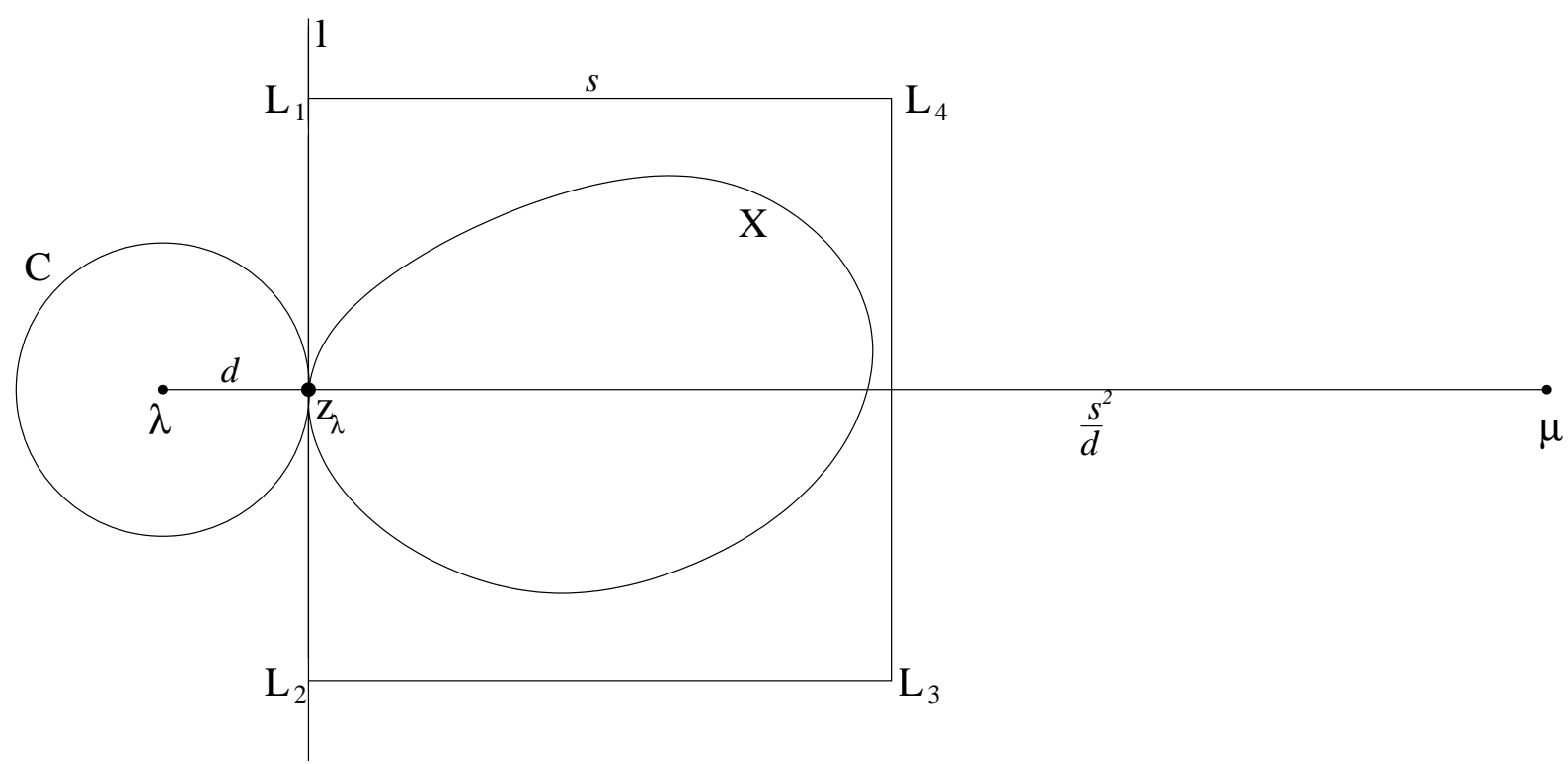

FiguRE 4 . We define $\mu$ to be the point on the half-line passing through the points $\lambda$ and $z_{\lambda}$ with initial point $\lambda$ such that $\mu$ is at distance $\frac{s^{2}}{d}$ from $z_{\lambda}$. Since $\frac{s^{2}}{d}>s$, $\mu$ lies outside the box $L_{1} L_{2} L_{3} L_{4}$ and therefore outside $X$. 
Let us show that $\mu$ also satisfies the other condition in the hypothesis, i.e.,

$$
|\lambda-\mu|>|z-\mu|
$$

for all $z$ in $X$.

Indeed, let $z$ be an arbitrary point in $X$ and let $z_{\mu}$ be its projection on the line passing through the points $z_{\lambda}$ and $\mu$. The distance from $z$ to $\mu$ is the length of the hypotenuse in the right triangle $\triangle z z_{\mu} \mu$ (see Figure 5).

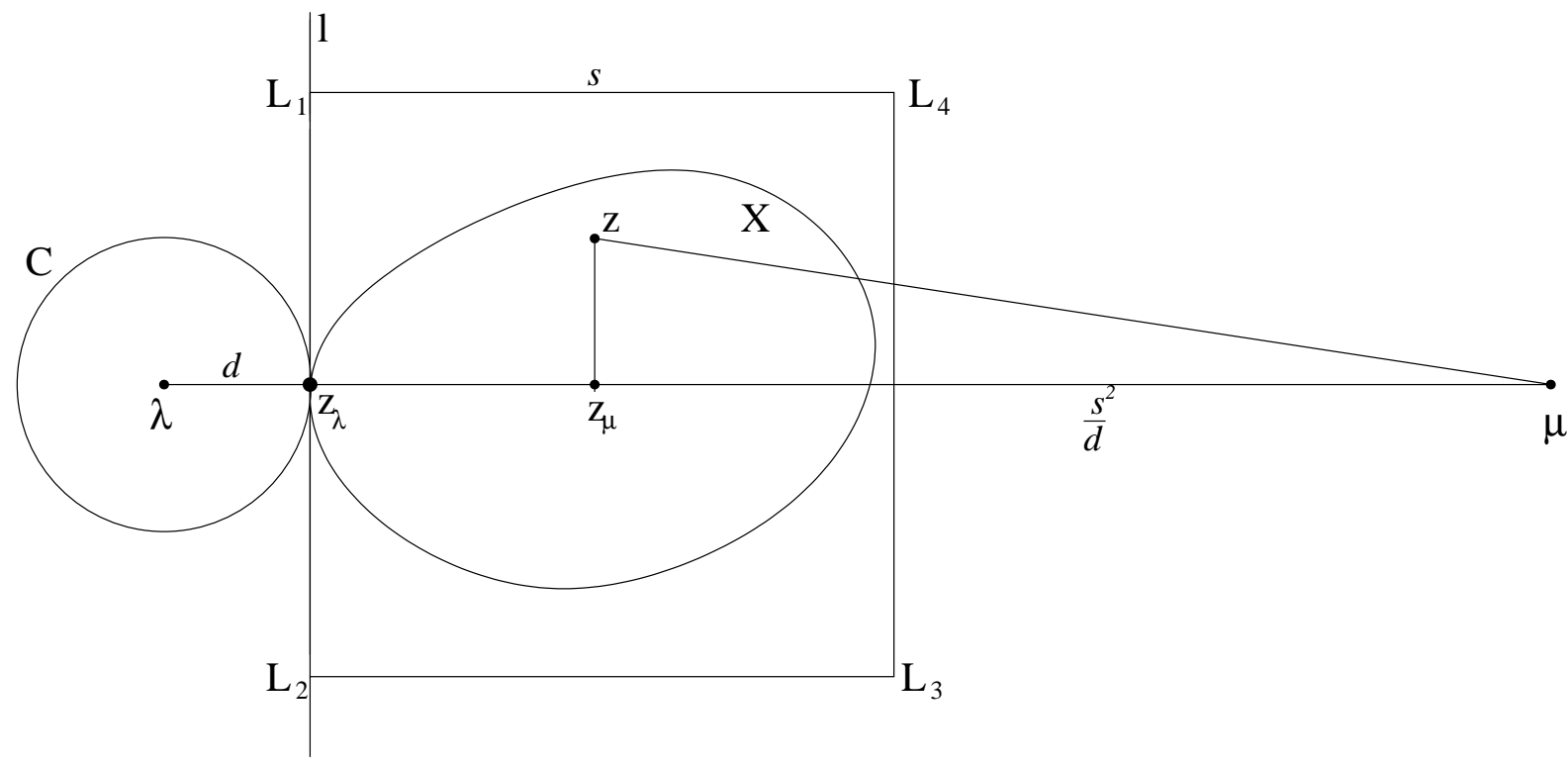

Figure 5. The distance from a point $z$ in $X$ to $\mu$ is the length of the hypotenuse in the right triangle $\triangle z z_{\mu} \mu$, where $z_{\mu}$ is the projection of $z$ on the line passing through the points $z_{\lambda}$ and $\mu$

Since $\mu$ lies outside the square $L_{1} L_{2} L_{3} L_{4}$, the point $z_{\mu}$ lies on the line segment $\left[z_{\lambda} \mu\right]$, hence

$$
\left|z_{\mu}-\mu\right| \leq\left|z_{\lambda}-\mu\right|
$$

Note that since $X$ is contained in the square $L_{1} L_{2} L_{3} L_{4}$ and the line $z_{\lambda} \mu$ passing through the points $z_{\lambda}$ and $\mu$ is perpendicular on the side $L_{1} L_{2}$, the projection $z_{\mu}$ of the point $z$ in $X$ on $z_{\lambda} \mu$ falls inside $L_{1} L_{2} L_{3} L_{4}$. Since the largest distance between any two points inside a square is the length of the diagonal, we have

$$
\left|z_{\mu}-z\right| \leq s \sqrt{2}
$$

By the Pythagorean Theorem and inequalities (3.6) and (3.7),

$$
|z-\mu|^{2}=\left|z_{\mu}-\mu\right|^{2}+\mid \begin{gathered}
z_{\mu}-\left.z\right|^{2} \leq\left|z_{\lambda}-\mu\right|^{2}+2 s^{2} \\
.
\end{gathered}
$$


On the other hand, by the equation defining $\mu$ (3.4) we have the following formula for the square of the length of the line segment $[\lambda \mu]$ :

$$
\begin{aligned}
|\lambda-\mu|^{2} & =\left(\left|\lambda-z_{\lambda}\right|+\left|z_{\lambda}-\mu\right|\right)^{2}=\left(d+\left|z_{\lambda}-\mu\right|\right)^{2} \\
& =d^{2}+2 d\left|z_{\lambda}-\mu\right|+\left|z_{\lambda}-\mu\right|^{2}=d^{2}+2 d \frac{s^{2}}{d}+\left|z_{\lambda}-\mu\right|^{2} \\
& =d^{2}+2 s^{2}+\left|z_{\lambda}-\mu\right|^{2} .
\end{aligned}
$$

Since $d$ is positive, by formula (3.11) we have

$$
|\lambda-\mu|^{2}=d^{2}+2 s^{2}+\left|z_{\lambda}-\mu\right|^{2}>2 s^{2}+\left|z_{\lambda}-\mu\right|^{2} .
$$

Combining inequalities (3.12) and (3.8) we obtain

$$
|\lambda-\mu|^{2}>|z-\mu|^{2} \text {. }
$$

Finally, taking the square root of both sides yields

$$
|\lambda-\mu|>|z-\mu| \text {. }
$$

Since $z$ in $X$ was chosen arbitrarily, inequality (3.14) holds for all $z$ in $X$, therefore $\mu$ is a point satisfying the conditions in the hypothesis.

3.2. Analytical Theorem. In the beginning of this section, we summarize a few wellknown facts in functional analysis, which are used in the proof of Theorem 3.2.

Let $X$ be a measurable subset of the complex plane. The linear space of square integrable complex functions on $X$ is generally denoted by $L^{2}(X)$. As is well-known, $L^{2}(X)$ has the structure of a complex Hilbert space with the inner product defined by

$$
(f, g)=\iint_{X} f(x, y) \overline{g(x, y)} d x d y \text {. }
$$

Let $U$ be an arbitrary finite-dimensional subspace of $L^{2}(X)$. We denote by $P_{U}$ the orthogonal projection $L^{2}(X) \longrightarrow U$ and by $T_{U}$ the operator multiplication by $x+i y$ on the space $U$ :

$$
\begin{aligned}
T_{U}: U & \longrightarrow L^{2}(X), \\
T_{U}(f(x, y)) & =(x+i y) \cdot f(x, y) .
\end{aligned}
$$

It is easy to see that $T_{U}$ is a linear operator on $U$, hence the composition $P_{U} \circ T_{U}$ is a linear operator on the finite-dimensional space $U$.

The following theorem lists a few basic properties of the orthogonal projection operator. A proof can be be found, for instance, in [1].

Theorem 3.1. Let $X$ be a measurable subset of $\mathbb{C}$ and $U$ be a nonzero finite-dimensional subspace of $L^{2}(X)$. The orthogonal projection $P_{U}$ has the following properties:

(1) $P_{U}$ is a linear operator with operator norm equal to 1.

(2) The restriction of $P_{U}$ to the space $U$ is the identity operator on $U$, i.e., $P_{U}(\varphi)=$ $\varphi$ for all functions $\varphi$ in $U$. 
(3) The operator $P_{U}$ is self-adjoint, i.e., for all $\varphi, \psi$ in $L^{2}(X)$,

$$
\left(P_{U}(\varphi), \psi\right)=\left(\varphi, P_{U}(\psi)\right) \text {. }
$$

The principal tool for the constructions of discretizations and quadratures in this paper is the following analytical theorem.

Theorem 3.2. Let $X$ be a measurable, convex, closed and bounded subset of $\mathbb{C}$, and $U$ be a finite-dimensional linear subspace of $L^{2}(X)$. Let $T_{U}$ be the linear operator defined by (3.16), and $P_{U}$ be the projection onto the space $U$. Then all the eigenvalues of the operator $P_{U} \circ T_{U}$ fall inside $X$.

Proof. The theorem is proved by contradiction. To this end, suppose that the operator $P_{U} \circ T_{U}$ has an eigenvalue $\lambda \in \mathbb{C}$ outside $X$. By Lemma 3.1, there exists $\mu \in \mathbb{C}$ such that $|\mu-\lambda|>|\mu-z|$, for all $z \in X$, hence

$$
|\mu-\lambda|>\max _{z \in X}|\mu-z| .
$$

Since $\lambda$ is an eigenvalue for $P_{U} \circ T_{U}$, there exists a nonzero $\varphi \in U$ such that

$$
P_{U}\left(T_{U}(\varphi)\right)=\lambda \varphi
$$

Subtracting $\mu \varphi$ from both sides of (3.19) we obtain

$$
P_{U}\left(T_{U}(\varphi)\right)-\mu \varphi=\lambda \varphi-\mu \varphi .
$$

Since $\varphi$ belongs to the subspace $U$, by Theorem 3.1, we have $\mu \varphi=P_{U}(\mu \varphi)$, therefore we can rewrite $(3.20)$ as

$$
P_{U}\left(T_{U}(\varphi)\right)-P_{U}(\mu \varphi)=\lambda \varphi-\mu \varphi,
$$

which is equivalent to

$$
P_{U}\left(T_{U}(\varphi)-\mu \varphi\right)=(\lambda-\mu) \varphi
$$

The operator norm of the projection $P_{U}$ is 1 by Theorem 3.1, therefore

$$
\left\|T_{U}(\varphi)-\mu \varphi\right\| \geq\left\|P_{U}\left(T_{U}(\varphi)-\mu \varphi\right)\right\| .
$$

Combining equation (3.22) with inequality (3.23) we obtain

$$
\left\|T_{U}(\varphi)-\mu \varphi\right\| \geq\|(\lambda-\mu) \varphi\| .
$$

Using the formula defining $T_{U}$ (3.16b), inequality (3.24) becomes

$$
\|(x+i y) \varphi(x, y)-\mu \varphi(x, y)\| \geq\|(\lambda-\mu) \varphi\|,
$$

which is equivalent to

$$
\|(x+i y-\mu) \varphi(x, y)\| \geq|\lambda-\mu| \cdot\|\varphi(x, y)\| .
$$

The complex variable $x+i y$ takes values only within the set $X$, therefore

$$
\left(\max _{z \in X}|z-\mu|\right) \cdot\|\varphi(x, y)\| \geq\|(x+i y-\mu) \varphi(x, y)\| \text {. }
$$


Inequalities (3.26) and (3.27) together yield

$$
\left(\max _{z \in X}|z-\mu|\right) \cdot\|\varphi(x, y)\| \geq|\lambda-\mu| \cdot\|\varphi(x, y)\| .
$$

However, $\varphi$ is nonzero, hence we can divide both sides of (3.28) by the positive number $\|\varphi(x, y)\|$ and obtain

$$
\max _{z \in X}|z-\mu| \geq|\lambda-\mu|
$$

inequality which contradicts our choice of $\mu$ (3.18).

\subsection{Remarks.}

Remark 3.1. The eigenvalues of the operator $P_{U} \circ T_{U}$ are complex numbers that fall inside $X$, hence they give us a discretization of the region $X$ that encapsulates information about the finite-dimensional space $U$. Theorem 3.2 provides a method for generating discretizations of (arbitrary) bounded convex regions in two dimensions. For a given region, one can vary the choice of the subspace $U$ in order to obtain different discretizations.

Remark 3.2. Let $G$ be the group of symmetries of $X$, i.e., $G$ consists of all isometries under which $X$ is invariant. Suppose that the subspace $U$ of $L^{2}(X)$ is invariant under the action of the group $G$, i.e.,

$$
g(f) \in U,
$$

for all $g \in G$ and $f \in U$, where the action of an isometry $g$ in $G$ on a function $f$ in $U$ is defined by

$$
\begin{aligned}
g(f) & =f_{g} \in L^{2}(X), \\
f_{g}(x, y) & =f\left(g^{-1}(x, y)\right), \text { for all }(x, y) \in X .
\end{aligned}
$$

Then the spectrum of the operator $P \circ T$ is also invariant under the action of $G$, i.e., if $\lambda$ is an eigenvalue of $P \circ T$, then $g(\lambda)$ is also an eigenvalue of $P \circ T$.

We can summarize this remark in the following way. If the subspace $U$ is chosen as to be invariant under the action of the symmetry group of $X$, then the discretization given by Theorem 3.2 will be symmetric (invariant under the symmetry group of $X$ ).

Remark 3.3. The nodes of the discretization given by Theorem 3.2, together with suitably chosen weights, provide a good initial point for Newton's method, which can be used to obtain a quadrature rule on $X$. This application of Theorem 3.2 is illustrated in Section 4.

Remark 3.4. Let $\left\{\varphi_{1}, \varphi_{2}, \ldots, \varphi_{n}\right\}$ be an orthonormal basis of $U$. The matrix $A$ representing $P_{U} \circ T_{U}$ in this basis has entries $\left(a_{i j}\right)$ defined by

$$
a_{i j}=\left(\left(P_{U} \circ T_{U}\right)\left(\varphi_{j}\right), \varphi_{i}\right) .
$$

By Theorem 3.1, the orthogonal projection operator is self-adjoint, hence

$$
a_{i j}=\left(T_{U}\left(\varphi_{j}\right), P_{U}\left(\varphi_{i}\right)\right) .
$$


Since $\varphi_{i}$ belongs to $U$, by Theorem 3.1, $P_{U}\left(\varphi_{i}\right)=\varphi_{i}$, for all $1 \leq i \leq n$. Therefore we can rewrite equation (3.34) as

$$
a_{i j}=\left(T_{U}\left(\varphi_{j}\right), \varphi_{i}\right) .
$$

Finally, we can use the definition of $T_{U}(3.16 \mathrm{~b})$ and of the inner product (3.15) to write equation (3.35) in the following explicit form:

$$
\begin{aligned}
a_{i j} & =\left(T_{U}\left(\varphi_{j}\right), \varphi_{i}\right) \\
& =\left((x+i y) \varphi_{j}(x, y), \varphi_{i}(x, y)\right) \\
& =\iint_{X}(x+i y) \cdot \varphi_{j}(x, y) \cdot \overline{\varphi_{i}(x, y)} d x d y .
\end{aligned}
$$

This explicit formula is used in Stage 3 of the algorithm described in the next section.

\section{Numerical Algorithm}

This section describes a numerical algorithm for the construction of a class of interpolation and quadrature formulae on regular polygons in the plane. The algorithm can be generalized to arbitrary bounded convex regions in $\mathbb{R}^{2}$.

For simplicity, we assume that the regular polygon is centered at the origin and its vertices lie on the unit circle. Let $s$ be the number of sides of the polygon $(s \geq 3)$, and $X$ be the convex region enclosed by the polygon. For a given positive integer $n$, we denote by $\mathcal{P}_{n}$ the space of polynomials on $X$ of total degree at most $n$ :

$$
\mathcal{P}_{n}=\{P(x, y): \text { total degree of } \mathrm{P} \leq n\} \subset L^{2}(X) .
$$

One can easily see that the dimension of $\mathcal{P}_{n}$ is given by the formula

$$
\operatorname{dim} \mathcal{P}_{n}=(n+1)(n+2) / 2 .
$$

The algorithm uses Theorem 3.2 with $U=\mathcal{P}_{n}, P: L^{2}(X) \longrightarrow U$ the orthogonal projection operator, and $T$ the multiplication by $(x+i y)$ operator (as defined by $(3.16 \mathrm{~b})$ ), in order to discretize the region $X$. Note that the space $\mathcal{P}_{n}$ is invariant under the action of the dihedral group $D_{s}$, the symmetry group of $X$. Therefore, by Remark 3.2 , the discretization given by the theorem is symmetric (invariant under the action of $D_{s}$ ). The nodes of this discretization are used as initial nodes for Newton's method in order to construct quadrature rules on the regular polygon $X$.

The algorithm's input is

$$
\begin{aligned}
& s=\text { the number of sides of the polygon } \\
& n=\text { the initial maximum degree of the polynomials } \\
& m=\text { the maximum degree of the polynomials to be integrated. }
\end{aligned}
$$

By equation (4.2), the dimensions of $\mathcal{P}_{n}$ and $\mathcal{P}_{m}$ are $N=(n+1)(n+2) / 2$ and $M=$ $(m+1)(m+2) / 2$, respectively. The algorithm's output is a quadrature rule on $X$ 
consisting of a set of $N$ nodes $\left(x_{1}, y_{1}\right),\left(x_{2}, y_{2}\right), \ldots,\left(x_{N}, y_{N}\right)$ and a set of $N$ weights $w_{1}, w_{2}, \ldots, w_{N}$ such that

$$
\iint_{X} \varphi(x, y) d x d y \sim \sum_{j=1}^{N} \varphi\left(x_{j}, y_{j}\right) w_{j}
$$

for any polynomial $\varphi$ in the $M$-dimensional space $\mathcal{P}_{m}$.

The algorithm proceeds in 4 stages.

In the first stage, we find a quadrature of the polygon by dividing it into triangles, and then constructing a tensor product of Gaussian quadratures on each of the triangles. In the second stage we construct an orthonormal basis for the space $\mathcal{P}_{n}$ iteratively, using the SVD. In the third stage we find the eigenvalues of the operator $P \circ T$. In the fourth stage we use Newton's method to modify the position of the eigenvalues and of the initial weights, found using the Least Squares Method, in order to obtain a quadrature rule which integrates all polynomials of total degree at most $m$.

\section{Stage 1: Discretize the polygon.}

In this stage we find a quadrature of the regular polygon by performing the following sequence of steps:

(1) Divide the regular polygon $A_{1} A_{2} \ldots A_{n}$ centered at $O$ into $n$ triangles $O A_{1} A_{2}, O A_{2} A_{3}, \ldots, O A_{n} A_{1}$, where $O$ is the origin of the axes.

(2) Discretize each of the triangles by constructing the tensor product of two onedimensional Gaussian quadratures on an interval. The order of the Gaussian quadratures is chosen sufficiently large so that the tensor product quadrature integrates all polynomials of total degree at most $m$ exactly.

(3) Assemble all the nodes and weights from all the triangles to obtain a quadrature of the polygon.

\section{Stage 2: Construct the orthonormal basis.}

In this stage, we find an orthonormal basis for the space $\mathcal{P}_{n}$. The basis is used for determining the eigenvalues of the operator $P_{\mathcal{P}_{n}} \circ T_{\mathcal{P}_{n}}=P \circ T$ on the space $\mathcal{P}_{n}$, therefore the choice of this basis is not important - any basis yields the same eigenvalues. The following algorithm for constructing an orthonormal basis is not optimal, however, it is stable and easy to implement.

(1) Consider the vector corresponding to the constant polynomial 1. Normalize it. The resulting vector provides an orthonormal basis for $\mathcal{P}_{0}$.

(2) Suppose we have constructed an orthonormal basis of $\mathcal{P}_{k}$, consisting of $K=$ $(k+1)(k+2) / 2$ vectors. Multiply each of the vectors in this basis by $x$ and $y$, respectively, and put the resulting vectors, together with the vectors in the basis of $\mathcal{P}_{k}$, in a matrix having $3 K$ columns. By performing SVD on this matrix we find an orthonormal basis for its column space, which is also an orthonormal basis for $\mathcal{P}_{k+1}$.

(3) Apply iteratively the procedure described in the previous step to obtain an orthonormal basis for $\mathcal{P}_{n}$. 


\section{Stage 3: Find the eigenvalues of $P \circ T$.}

(1) Compute the entries $\left(a_{i j}\right)$ of the matrix $A$ representing $P \circ T$ in the basis $\left\{\varphi_{1}, \varphi_{2}, \ldots, \varphi_{N}\right\}$ constructed in Stage 2 using Formula (3.38):

$$
a_{i j}=\iint_{X}(x+i y) \varphi_{i}(x, y) \overline{\varphi_{j}(x, y)} d x d y .
$$

The integrals are evaluated using the quadrature constructed in Stage 1.

(2) Find the eigenvalues $\lambda_{1}, \lambda_{2}, \ldots, \lambda_{N}$ of the complex matrix $A$ using the QR algorithm described in Section 2.2.

\section{Stage 4: Construct the quadrature.}

In this stage, we find a quadrature rule consisting of $N$ nodes and $N$ weights which integrates all $M$ polynomials of total degree at most $m$. The algorithm uses Newton's method and consists of the following sequence of steps:

(1) Construct an orthonormal basis $\varphi_{1}, \varphi_{2}, \ldots, \varphi_{M}$ of the space $\mathcal{P}_{m}$ using the procedure described in Stage 2.

(2) The initial quadrature points $\left(x_{1}^{0}, y_{1}^{0}\right),\left(x_{2}^{0}, y_{2}^{0}\right), \ldots,\left(x_{N}^{0}, y_{N}^{0}\right)$ for Newton's method are given by the eigenvalues:

$$
x_{j}^{0}+i y_{j}^{0}=\lambda_{j}, \quad j=1,2, \ldots, N .
$$

The initial quadrature weights $w_{1}^{0}, w_{2}^{0}, \ldots, w_{N}^{0}$ are found by solving the following linear system using Least Squares:

$\varphi_{j}\left(x_{1}^{0}, y_{1}^{0}\right) w_{1}^{0}+\varphi_{j}\left(x_{2}^{0}, y_{2}^{0}\right) w_{2}^{0}+\ldots+\varphi_{j}\left(x_{N}^{0}, y_{N}^{0}\right) w_{N}^{0}=I_{j}, \quad j=1,2, \ldots, M$,

where

$$
I_{j}=\iint_{X} \varphi_{j}(x, y) d x d y, \quad j=1,2, \ldots, M
$$

are the exact values of the integrals of the polynomials in the orthonormal basis of $\mathcal{P}_{m}$ found in step (1). The integrals $I_{j}, j=1,2, \ldots, M$ are found using the initial quadrature constructed in Stage 1.

(3) Find a quadrature rule for integrating all polynomials in $\mathcal{P}_{m}$. This is equivalent to solving the following nonlinear system with $3 N$ unknowns

$$
\left(w_{1}, w_{2}, \ldots, w_{N}, x_{1}, x_{2}, \ldots, x_{N}, y_{1}, y_{2}, \ldots, y_{N}\right)
$$

and $M$ equations:

$$
\varphi_{j}\left(x_{1}, y_{1}\right) w_{1}+\varphi_{j}\left(x_{2}, y_{2}\right) w_{2}+\ldots+\varphi_{j}\left(x_{N}, y_{N}\right) w_{N}=I_{j}, \quad j=1,2, \ldots, M .
$$

Finding a solution to this system is equivalent to finding a zero of the smooth function $F: \mathbb{R}^{3 N} \longrightarrow \mathbb{R}^{M}$ defined by

$$
F\left(w_{1}, \ldots, w_{N}, x_{1}, \ldots, x_{N}, y_{1}, \ldots, y_{N}\right):=\left(\begin{array}{c}
\sum_{i=1}^{N} \varphi_{1}\left(x_{i}, y_{i}\right) w_{i}-I_{1} \\
\ldots \\
\sum_{i=1}^{N} \varphi_{M}\left(x_{i}, y_{i}\right) w_{i}-I_{M}
\end{array}\right) \text {. }
$$


In order to find a zero of $F$, we use Least Squares Newton's method (as described in Section 2.3) with initial point

$$
\left(w_{1}^{0}, w_{2}^{0}, \ldots, w_{N}^{0}, x_{1}^{0}, x_{2}^{0}, \ldots, x_{N}^{0}, y_{1}^{0}, y_{2}^{0}, \ldots, y_{N}^{0}\right) .
$$

The weights and nodes given by this zero of $F$ define an $N$-point quadrature which integrates all polynomials in the $M$-dimensional space $\mathcal{P}_{m}$.

\section{Numerical Results}

We implemented the procedure described in Section 4 and tested it on a number of examples. Several of the results are listed below, in order to demonstrate the performance of the algorithm. The algorithm was implemented in FORTRAN and compiled with the Lahey-Fujitsu FORTRAN 95 compiler. Computations were performed in double and extended precision arithmetic.

5.1. Quadratures and interpolation formulae on the triangle. In this section we describe the quadratures and interpolation formulae on an equilateral triangle generated by the algorithm. The triangle is centered at the origin and has vertices located at the points $\left\{1, e^{2 \pi / 3}, e^{4 \pi / 3}\right\}$ on the unit circle. The following procedure is used.

We begin with an initial polynomial degree $n$. Using the algorithm described in Section 4 , we find the maximum polynomial degree $m$ for which Least Squares Newton's method yields a quadrature having positive weights. The resulting quadratures have $N=(n+1)(n+2) / 2$ nodes and can integrate all the polynomials in the space $\mathcal{P}_{m}$ of dimension $M=(m+1)(m+2) / 2$.

Remark 5.1. Given the orthonormal basis $\varphi_{1}, \varphi_{2}, \ldots, \varphi_{N}$ of the space $\mathcal{P}_{n}$ constructed in Stage 2 of the algorithm, the $N$ nodes $x_{1}, x_{2}, \ldots, x_{N}$ of the quadrature yield an interpolation formula relative to the functions $\left\{\varphi_{i}\right\}$. The number of nodes for this interpolation formula equals the number of functions. The formula is stable if the condition number of the associated interpolation matrix $B$ is small, where $B$ is defined by the equations

$$
B=\left(b_{i j}\right), \quad b_{i j}=\varphi_{i}\left(x_{j}\right), \quad i, j=1,2, \ldots, N .
$$

In Tables 1 and 2 we list the quadratures generated by this procedure, together with the corresponding efficiency coefficients $e$ (as defined by Equation 2.9) and condition numbers of the interpolation matrices $B$ (as defined by Equation 5.1). The quadratures of degrees 9, 11, 16, 21, 26, 29 and 31 have rotational symmetry, but not reflection symmetry, while all the remaining quadratures are fully symmetric — cf. Remark 5.2.

Remark 5.2. In most cases, since the eigenvalues are invariant under the action of the symmetry group of the triangle, modifying their position by Least Squares Newton's method yields quadratures on the triangle which are fully symmetric. In a few instances, however, relaxing the requirement that the quadratures have full symmetry, and allowing only rotational symmetry instead, allows us to obtain quadratures for higher orders $m$. The phenomenon has not been investigated further. 


\begin{tabular}{|c||c|c|c|c|c|c|c|c|c|c|}
\hline $\mathrm{n}$ & 1 & 2 & 3 & 4 & 5 & 6 & 7 & 8 & 9 & 10 \\
$\mathrm{~m}$ & 2 & 4 & 5 & 7 & 9 & 11 & 12 & 14 & 16 & 17 \\
\hline $\mathrm{N}$ & 3 & 6 & 10 & 15 & 21 & 28 & 36 & 45 & 55 & 66 \\
$\mathrm{M}$ & 6 & 15 & 21 & 36 & 55 & 78 & 91 & 120 & 153 & 171 \\
\hline $\mathrm{e}$ & 0.67 & 0.83 & 0.70 & 0.80 & 0.87 & 0.93 & 0.84 & 0.89 & 0.93 & 0.86 \\
\hline \hline $\mathrm{CN}$ & 1.0 & 1.0 & 1.2 & 1.4 & 1.6 & 2.1 & 1.8 & 2.3 & 4.2 & 5.9 \\
\hline
\end{tabular}

TABLE 1. Triangle quadratures - part 1. For each initial polynomial degree $n$ from 1 to 10 , we list the corresponding maximum degree $m$ of a quadrature having positive weights. The number of nodes of the quadrature is $N=(n+1)(n+2) / 2$ and the number of polynomials which can be integrated exactly is $M=\operatorname{dim} \mathcal{P}_{m}=(m+1)(m+2) / 2$. The efficiency of the quadrature is $e=M /(3 N)$. The condition number of the interpolation matrix (5.1) is listed in the row labeled $C N$.

\begin{tabular}{|c||c|c|c|c|c|c|c|c|c|}
\hline $\mathrm{n}$ & 11 & 12 & 13 & 14 & 15 & 16 & 17 & 18 & 19 \\
$\mathrm{~m}$ & 19 & 21 & 22 & 23 & 26 & 27 & 29 & 31 & 32 \\
\hline $\mathrm{N}$ & 78 & 91 & 105 & 120 & 136 & 153 & 171 & 190 & 210 \\
$\mathrm{M}$ & 210 & 253 & 276 & 300 & 378 & 406 & 465 & 528 & 561 \\
\hline $\mathrm{e}$ & 0.90 & 0.93 & 0.88 & 0.83 & 0.93 & 0.88 & 0.91 & 0.93 & 0.89 \\
\hline \hline $\mathrm{CN}$ & 5.0 & 9.7 & 6.3 & 13.1 & 68.6 & 144.0 & 67.8 & 71.5 & 56.2 \\
\hline
\end{tabular}

TABLE 2. Triangle quadratures - part 2. For each initial polynomial degree $n$ from 11 to 19 , we list the corresponding maximum degree $m$ of a quadrature having positive weights. The number of nodes of the quadrature is $N=(n+1)(n+2) / 2$ and the number of polynomials which can be integrated exactly is $M=\operatorname{dim} \mathcal{P}_{m}=(m+1)(m+2) / 2$. The efficiency of the quadrature is $e=M /(3 N)$. The condition number of the interpolation matrix (5.1) is listed in the row labeled $C N$.

In Tables 3 and 4, we list two of the quadrature rules on the triangle obtained using the procedure described above. Since each of the quadratures has full symmetry, only a subset of generating nodes and corresponding weights is sufficient for describing each of the quadratures (see, for example, [11] for more details). The remaining nodes may be obtained via the action of the symmetry group of the triangle, with the obvious observation that points belonging to the same orbit have the same weight. The quadratures were computed in extended precision and are listed with 20 digits.

In Figure 6, we show a picture of the nodes of the fully symmetric quadrature of order 32 .

5.2. Quadratures on other polygons. Constructing quadratures on regular polygons having at least 4 sides using the algorithm described in Section 4 is slightly more 


\begin{tabular}{|c|c|c|}
\hline $\mathrm{x}$ & $\mathrm{y}$ & Weight \\
\hline$-.16868265317233866793 \mathrm{E}+00$ & $0.29216692565001003106 \mathrm{E}+00$ & $0.58935620335540765011 \mathrm{E}-01$ \\
$-.15808488336403957216 \mathrm{E}+00$ & $-.12519480877035419803 \mathrm{E}-30$ & $0.67452219369552326397 \mathrm{E}-01$ \\
$-.35347157488571107334 \mathrm{E}+00$ & $0.14203631281845219952 \mathrm{E}+00$ & $0.44503540566573551812 \mathrm{E}-01$ \\
$-.47009724117048709338 \mathrm{E}+00$ & $0.41794071711619191254 \mathrm{E}-30$ & $0.17897106324896028214 \mathrm{E}-01$ \\
$-.36136906345529792533 \mathrm{E}+00$ & $0.40253738576591622170 \mathrm{E}+00$ & $0.36144179089957608755 \mathrm{E}-01$ \\
$-.47259519998535818893 \mathrm{E}+00$ & $0.24682585755277199100 \mathrm{E}+00$ & $0.18293541893943411802 \mathrm{E}-01$ \\
$-.47234427519430125315 \mathrm{E}+00$ & $0.50374523862970667221 \mathrm{E}+00$ & $0.16917794610623270344 \mathrm{E}-01$ \\
$-.35112713454811437607 \mathrm{E}+00$ & $0.60817003695340733938 \mathrm{E}+00$ & $0.28921171272990801675 \mathrm{E}-01$ \\
$-.47085943420511108264 \mathrm{E}+00$ & $0.70900463999219136033 \mathrm{E}+00$ & $0.12279549423359387150 \mathrm{E}-01$ \\
$-.47949927002953977850 \mathrm{E}+00$ & $0.83051709788335153417 \mathrm{E}+00$ & $0.35293734203249423613 \mathrm{E}-02$ \\
\hline
\end{tabular}

TABLE 3. A fully symmetric 45-point quadrature of order 14 on the triangle. Only 10 generating nodes are listed; the remaining nodes may be obtained via the action of the group of symmetries of the triangle.

\begin{tabular}{|c|c|c|}
\hline $\mathrm{x}$ & $\mathrm{y}$ & Weight \\
\hline$-.12399257021092653653 \mathrm{E}+00$ & $0.19374825509442976738 \mathrm{E}-28$ & $0.41450918407684045121 \mathrm{E}-01$ \\
$-.13177700392715291090 \mathrm{E}+00$ & $0.22824446607103231387 \mathrm{E}+00$ & $0.38440468676165286217 \mathrm{E}-01$ \\
$-.28778371647987152368 \mathrm{E}+00$ & $0.10998294723245009451 \mathrm{E}+00$ & $0.31317527185910258616 \mathrm{E}-01$ \\
$-.40630672781840910415 \mathrm{E}+00$ & $-.14456488256121493556 \mathrm{E}-28$ & $0.14002753888728943368 \mathrm{E}-01$ \\
$-.29724898552695112330 \mathrm{E}+00$ & $0.31498865266126179066 \mathrm{E}+00$ & $0.26827201883108899153 \mathrm{E}-01$ \\
$-.41356124787667862272 \mathrm{E}+00$ & $0.16719641141490991682 \mathrm{E}+00$ & $0.19961899945473734702 \mathrm{E}-01$ \\
$-.48264071535159024307 \mathrm{E}+00$ & $0.95221898752783636336 \mathrm{E}-01$ & $0.86539518883907294556 \mathrm{E}-02$ \\
$-.41408886151213149256 \mathrm{E}+00$ & $0.38336091989130268782 \mathrm{E}+00$ & $0.20240988214359192248 \mathrm{E}-01$ \\
$-.27842964300896413291 \mathrm{E}+00$ & $0.48225428802479053639 \mathrm{E}+00$ & $0.23989433658357479662 \mathrm{E}-01$ \\
$-.48370152343347647465 \mathrm{E}+00$ & $0.30142300793320924164 \mathrm{E}+00$ & $0.87991644027906763838 \mathrm{E}-02$ \\
$-.48281402069237875749 \mathrm{E}+00$ & $0.49942229259718307221 \mathrm{E}+00$ & $0.78178774263925103734 \mathrm{E}-02$ \\
$-.40528714204595318336 \mathrm{E}+00$ & $0.57394856816236337313 \mathrm{E}+00$ & $0.18008780871442209707 \mathrm{E}-01$ \\
$-.48105533677985120342 \mathrm{E}+00$ & $0.64968083098649002499 \mathrm{E}+00$ & $0.60973338938233607918 \mathrm{E}-02$ \\
$-.41966365447253047169 \mathrm{E}+00$ & $0.72687877163645268678 \mathrm{E}+00$ & $0.10547116649857438346 \mathrm{E}-01$ \\
$-.48630312842497788637 \mathrm{E}+00$ & $0.76255697108968090949 \mathrm{E}+00$ & $0.34615238341665281813 \mathrm{E}-02$ \\
$-.48251983199382645129 \mathrm{E}+00$ & $0.83574886467290610008 \mathrm{E}+00$ & $0.22095115197099314446 \mathrm{E}-02$ \\
\hline
\end{tabular}

TABLE 4. A fully symmetric 78-point quadrature of order 19 on the triangle. Only 16 generating nodes are listed; the remaining nodes may be obtained via the action of the group of symmetries of the triangle.

involved. In contrast to the triangle case, the operator $P \circ T$ has sometimes multiple eigenvalues; in all cases, the only multiple eigenvalue is 0 , the center of the polygon.

It is easily seen that, in some cases, 0 is a multiple eigenvalue of the operator $P \circ T$ via the following argument.

Observation 5.1. Suppose that the number $N$ of eigenvalues is not divisible by the number of sides s. By Remark 3.2, the set of eigenvalues is invariant under rotations, hence we can divide the nonzero eigenvalues into $s$ groups, each corresponding to one of the sides of the polygon. Consequently, there are $s \cdot k$ nonzero eigenvalues, where $k$ is a nonnegative integer, and all the remaining $N-s \cdot k$ eigenvalues must be equal to 0 .

We used the procedure described in the first three stages of the algorithm in Section 4 to find the number of eigenvalues equal to 0 for all values of $s, 3 \leq s \leq 20$, and all values of $n, 1 \leq n \leq 26$, where $s$ is the number of sides of the regular polygon and $n$ is 


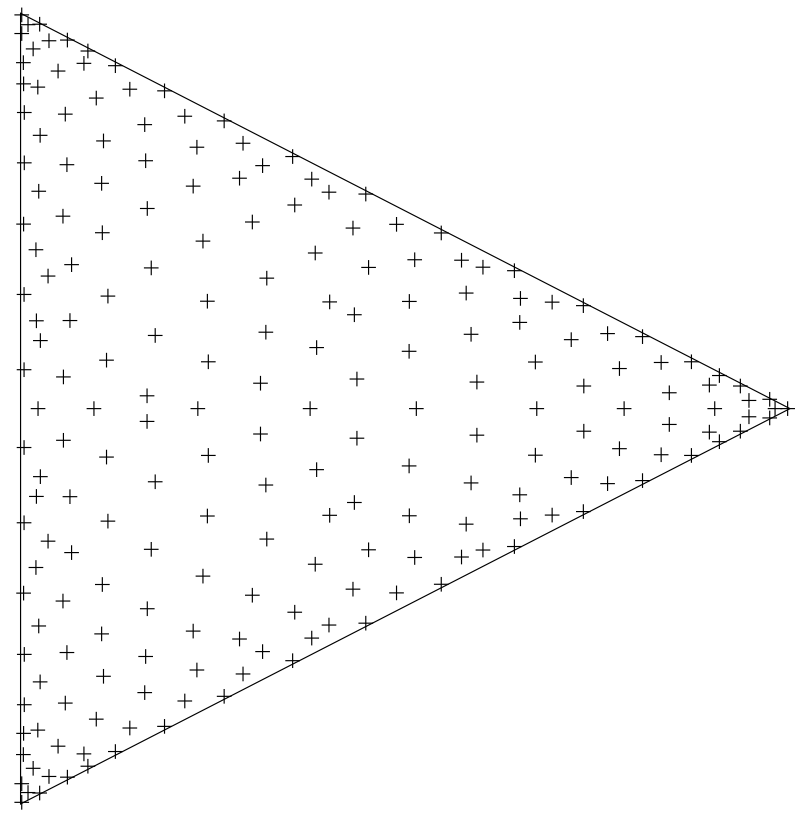

Figure 6. Symmetric quadrature of order 32 on the triangle.

the maximum degree of the polynomials in the space $U=\mathcal{P}_{n}$. The results are listed in Table 5 .

We note that the entries in the table seem to follow a certain pattern and, in fact, they can be described by the following formulae.

Conjecture 5.1. Let $s$ be the number of sides of the regular polygon $X$ centered at 0 and having all the vertices on the unit circle, and let $U=\mathcal{P}_{n}$ be the space of all polynomials on $X$ of total degree at most $n$. We denote by $\Lambda(n, s)$ the number of eigenvalues equal to 0 of the operator $P_{U} \circ T_{U}$, where the operator $P_{U} \circ T_{U}$ is defined as in the statement of Theorem 3.2.

Let us denote by $\mathcal{S}_{p}$ the sum of the first $p$ positive integers:

$$
\mathcal{S}_{p}=1+2+\ldots+p=p(p+1) / 2,
$$

with the convention that $\mathcal{S}_{-1}=\mathcal{S}_{0}=0$. Then, according to the parity of the number of sides $s$, one of the following formulae holds.

If $s=2 k+1$, where $k$ is a positive integer, then

$$
\Lambda(n, s)= \begin{cases}\mathcal{S}_{n(\bmod s)+1,} & \text { if } n(\bmod s) \leq k-1 \\ \mathcal{S}_{s-n(\bmod s)-2}, & \text { if } n(\bmod s) \geq k\end{cases}
$$

If $s=2 k$, where $k$ is an integer greater than 1 , then

$$
\Lambda(n, s)= \begin{cases}\mathcal{S}_{n(\bmod s)+1}+\frac{1}{2}(n-n(\bmod s)), & \text { if } n(\bmod s) \leq k-1 \\ \mathcal{S}_{s-n(\bmod s)-2}+\frac{1}{2}(n-n(\bmod s)+s), & \text { if } n(\bmod s) \geq k\end{cases}
$$

It can be easily checked that the conjecture holds for all values of $\Lambda(n, s)$ listed in Table 5. A proof for the general case is beyond the scope of this paper. 
Constructing quadratures in the cases when the operator $P \circ T$ has multiple zero eigenvalues is more involved because the condition number of the interpolation matrix (5.1) explodes. In several of these cases, Newton's method fails to find quadratures having positive weights. We illustrate the situation in Table 6. A dash denotes that no quadrature having positive weights was found, while a numbered entry denotes that quadratures having positive weights were found and the numbered entry is the highest order of such a quadrature.

\section{REFERENCES}

[1] Conway, John B., A Course in Functional Analysis, 2nd edition, Springer-Verlag, 1994.

[2] Cools, R., Constructing cubature formulae: The science behind the art, Acta Numerica, Vol. 6, Cambridge University Press, Cambridge, 1997, pp. 1-54.

[3] Dahlquist, G. And Bü̈RK, A., Numerical Methods, Dover, Mineola, NY, 2003.

[4] Davis, P. J., Rabinowitz, P., Methods of Numerical Integration, Academic Press, New York, 1984.

[5] Golub, V. H., Van LoAn, C. H., Matrix Computations, Johns Hopkins University Press, Baltimore, 1983.

[6] Isaacson, E. And Keller, H. B., Analysis of numerical Methods, John Wiley\&Sons, New York, 1966, and Dover, 2004.

[7] Stroud, A. H., Approximate calculation of multiple integrals, Prentice-Hall, Englewood Cliffs, NJ, 1971.

[8] Ma, J., Rokhlin, V., Wandzura, S., Generalized Gaussian quadrature of systems of arbitrary functions, SIAM J. Numer. Anal. 33 (3) (1996), pp. 971996.

[9] Martinsson, P.G., Rokhlin, V., Tygert, M., On Interpolation and Integration in FiniteDimensional Spaces of Bounded Functions, Communications in Applied Mathematics and Computational Science (CAMCoS), V. 1, pp. 133-142 (2006).

[10] Stoer, J., Bulirsch, R., Introduction To Numerical Analysis, 2nd edition, Springer-Verlag, 1992.

[11] Xiao, H., Gimbutas, Z., A numerical algorithm for the construction of efficient quadrature rules in two and higher dimensions, in: Computers and Mathematics with Applications 59(2010), pp. 663-676.

[12] Yarvin, N., Rokhlin, V., Generalized Gaussian quadratures and singular value decompositions of integral operators, SIAM J. Sci. Comput. 20 (2) (1998), pp. 669718. 


\begin{tabular}{|c|c|c|c|c|c|c|c|c|c|c|c|c|c|c|c|c|c|c|}
\hline & 3 & 4 & 5 & 6 & 7 & 8 & 9 & 10 & 11 & 12 & 13 & 14 & 15 & 16 & 17 & 18 & 19 & 20 \\
\hline 1 & 0 & 3 & 3 & 3 & 3 & 3 & 3 & 3 & 3 & 3 & 3 & 3 & 3 & 3 & 3 & 3 & 3 & 3 \\
\hline 2 & 0 & 2 & 1 & 6 & 6 & 6 & 6 & 6 & 6 & 6 & 6 & 6 & 6 & 6 & 6 & 6 & 6 & 6 \\
\hline 3 & 1 & 2 & 0 & 4 & 3 & 10 & 10 & 10 & 10 & 10 & 10 & 10 & 10 & 10 & 10 & 10 & 10 & 10 \\
\hline 4 & 0 & 3 & 0 & 3 & 1 & 7 & 6 & 15 & 15 & 15 & 15 & 15 & 15 & 15 & 15 & 15 & 15 & 15 \\
\hline 5 & 0 & 5 & 1 & 3 & 0 & 5 & 3 & 11 & 10 & 21 & 21 & 21 & 21 & 21 & 21 & 21 & 21 & 21 \\
\hline 6 & 1 & 4 & 3 & 4 & 0 & 4 & 1 & 8 & 6 & 16 & 15 & 28 & 28 & 28 & 28 & 28 & 28 & 28 \\
\hline 7 & 0 & 4 & 1 & 6 & 1 & 4 & 0 & 6 & 3 & 12 & 10 & 22 & 21 & 36 & 36 & 36 & 36 & 36 \\
\hline 8 & 0 & 5 & 0 & 9 & 3 & 5 & 0 & 5 & 1 & 9 & 6 & 17 & 15 & 29 & 28 & 45 & 45 & 45 \\
\hline 9 & 1 & 7 & 0 & 7 & 6 & 7 & 1 & 5 & 0 & 7 & 3 & 13 & 10 & 23 & 21 & 37 & 36 & 55 \\
\hline 10 & 0 & 6 & 1 & 6 & 3 & 10 & 3 & 6 & 0 & 6 & 1 & 10 & 6 & 18 & 15 & 30 & 28 & 46 \\
\hline 11 & 0 & 6 & 3 & 6 & 1 & 14 & 6 & 8 & 1 & 6 & 0 & 8 & 3 & 14 & 10 & 24 & 21 & 38 \\
\hline 12 & 1 & 7 & 1 & 7 & 0 & 11 & 10 & 11 & 3 & 7 & 0 & 7 & 1 & 11 & 6 & 19 & 15 & 31 \\
\hline 13 & 0 & 9 & 0 & 9 & 0 & 9 & 6 & 15 & 6 & 9 & 1 & 7 & 0 & 9 & 3 & 15 & 10 & 25 \\
\hline 14 & 0 & 8 & 0 & 12 & 1 & 8 & 3 & 20 & 10 & 12 & 3 & 8 & 0 & 8 & 1 & 12 & 6 & 20 \\
\hline 15 & 1 & 8 & 1 & 10 & 3 & 8 & 1 & 16 & 15 & 16 & 6 & 10 & 1 & 8 & 0 & 10 & 3 & 16 \\
\hline 16 & 0 & 9 & 3 & 9 & 6 & 9 & 0 & 13 & 10 & 21 & 10 & 13 & 3 & 9 & 0 & 9 & 1 & 13 \\
\hline 17 & 0 & 11 & 1 & 9 & 3 & 11 & 0 & 11 & 6 & 27 & 15 & 17 & 6 & 11 & 1 & 9 & 0 & 11 \\
\hline 18 & 1 & 10 & 0 & 10 & 1 & 14 & 1 & 10 & 3 & 22 & 21 & 22 & 10 & 14 & 3 & 10 & 0 & 10 \\
\hline 19 & 0 & 10 & 0 & 12 & 0 & 18 & 3 & 10 & 1 & 18 & 15 & 28 & 15 & 18 & 6 & 12 & 1 & 10 \\
\hline 20 & 0 & 11 & 1 & 15 & 0 & 15 & 6 & 11 & 0 & 15 & 10 & 35 & 21 & 23 & 10 & 15 & 3 & 11 \\
\hline 21 & 1 & 13 & 3 & 13 & 1 & 13 & 10 & 13 & 0 & 13 & 6 & 29 & 28 & 29 & 15 & 19 & 6 & 13 \\
\hline 22 & 0 & 12 & 1 & 12 & 3 & 12 & 6 & 16 & 1 & 12 & 3 & 24 & 21 & 36 & 21 & 24 & 10 & 16 \\
\hline 23 & 0 & 12 & 0 & 12 & 6 & 12 & 3 & 20 & 3 & 12 & 1 & 20 & 15 & 44 & 28 & 30 & 15 & 20 \\
\hline 24 & 1 & 13 & 0 & 13 & 3 & 13 & 1 & 25 & 6 & 13 & 0 & 17 & 10 & 37 & 36 & 37 & 21 & 25 \\
\hline 25 & 0 & 15 & 1 & 15 & 1 & 15 & 0 & 21 & 10 & 15 & 0 & 15 & 6 & 31 & 28 & 45 & 28 & 31 \\
\hline 26 & 0 & 14 & 3 & 18 & 0 & 18 & 0 & 18 & 15 & 18 & 1 & 14 & 3 & 26 & 21 & 54 & 36 & 38 \\
\hline
\end{tabular}

TABLE 5. The number of eigenvalues equal to zero for all the values of the number of sides from 3 to 20 (listed in the top row) and of the maximum degree of polynomials from 1 to 26 (listed in the first column). 


\begin{tabular}{|c||c|c|c|c|c|c|c|c|}
\hline & 3 & 4 & 5 & 6 & 7 & 8 & 9 & 10 \\
\hline \hline Square & 5 & 5 & - & 9 & - & 11 & - & 13 \\
\hline Pentagon & 4 & 7 & 6 & 9 & 10 & 12 & 11 & \\
\hline Hexagon & - & 7 & - & 7 & - & - & - & 11 \\
\hline Heptagon & 5 & 6 & 8 & 10 & 8 & 12 & - & - \\
\hline Octagon & - & - & 6 & 9 & 9 & 13 & - & - \\
\hline Nonagon & - & - & 8 & 10 & 11 & 13 & 14 & 14 \\
\hline Decagon & - & - & - & - & 9 & 11 & 13 & 11 \\
\hline
\end{tabular}

TABLE 6. Quadratures on regular polygons with at least 4 sides. The top row lists the initial maximum degree of polynomials $n$. A numbered entry denotes the maximum polynomial degree $m$ for which a quadrature having positive weights was found. A dash denotes that no quadrature having positive weights was found. 